\title{
Application of the DSC and spectroscopy methods in the analysis of the protective effect of extracts from the blueberry fruit of the genus Vaccinium in relation to the lipid membrane
}

\author{
Hanna Pruchnik ${ }^{1}$ D $\cdot$ Dorota Bonarska-Kujawa $^{1} \cdot$ Romuald Żyłka $^{1} \cdot J_{a n}$ Oszmiański ${ }^{2} \cdot$ Halina Kleszczyńska $^{1}$
}

Received: 1 November 2017/ Accepted: 21 June 2018/Published online: 30 June 2018

(c) The Author(s) 2018

\begin{abstract}
The aim of the study was to determine changes incurred in lipid model membranes by polyphenol compounds from blueberry fruit of the genus Vaccinium that includes low, high and black blueberry. In particular, the effect of extracts on the packing order in the lipid hydrophilic phase, the fluidity of the hydrophobic phase, as well as the temperature of the phase transition in phospholipids was studied. Model membranes were formed of dipalmitoylphosphatidylcholine (DPPC) and egg phosphatidylcholine. The interaction of extracts with lipids was studied using the differential scanning calorimetry, infrared spectroscopy (ATR-FTIR) and fluorimetry using Laurdan, Prodan and DPH probes. All experimental results indicate that the biggest changes are in the hydrophilic part of the lipid bilayer. The polyphenol compounds had practically no influence on fluidity in the hydrophobic region of the membranes. No changes in the temperature of the main phase transition of DPPC were observed and only a small change in the temperature of pretransition for high concentration of compounds. Results obtained with the ATR-FTIR method did not reveal any changes in the alkyl chain region of bilayer; however, a small shift of bands was observed for phosphate and choline groups-the broadest effect appeared for the wild bilberry. The findings indicate that polyphenolic compounds contained in extracts of blueberries interact with the membrane surface and therefore can protect it.
\end{abstract}

Keywords Blueberry extracts · Liposomes · Phase transition · DSC · Fluorimetry · ATR-FTIR

\section{Introduction}

Blueberry fruit of the genus Vaccinium, which includes low (Vaccinium angustifolium L.), high (Vaccinium corymbosum L.) and black blueberry (Vaccinium myrtillus L.), is consumed because of its taste and healing effect. Bilberry, for example, has historically been used as a medicinal herb to treat diarrhea and improve night vision. Analysis of bilberry fruits' composition shows that they are a rich

Hanna Pruchnik and Dorota Bonarska-Kujawaa have contributed equally to this work.

Hanna Pruchnik

hanna.pruchnik@upwr.edu.pl

1 Department of Physics and Biophysics, Wrocław University of Environmental and Life Sciences, Wrocław, Poland

2 Department of Fruit, Vegetable and Plant Nutraceutical Technology, Wrocław University of Environmental and Life Sciences, Wrocław, Poland source of nutraceutics like phenolic substances, carotenoids and vitamins [1-15].

Nowadays, there are very important endeavors to find new effective substances that would help in prevention and treatment of some dangerous diseases. Rational use of the substances in protection and treatment is possible only if, in addition to studies showing the health-enhancing properties of plant extracts and polyphenolic compounds, we understand the mechanism of their interaction with the living cell, with regard to possible side effects [16-18].

Lipids organization in the cell membrane guarantees integrity and functionality of the membrane. Changes in the lipid organization indicate changes in the physicochemical properties of the cell lipid bilayer membrane by, e.g., reduction in hydrophobicity of the lipid membrane interior, change in organization and fluidity of the bilayer and disorders of the lipid asymmetry and depolarization of the membrane [19-21]. As a consequence of those changes, an inhibition of the activity of the enzyme and transport of proteins and loss of membrane integrity may occur, leading 
to cell's death. Any biologically active substance influences the living organism in the first place through interaction with the cell membrane, which is the main and sometimes the only site where various physicochemical factors affect the body. Knowledge of the mechanisms responsible for the effects of polyphenolic compounds on organisms at the molecular level is of vital importance from the medical, dietary and related technologies point of view. It should be emphasized that until now the mechanism of interaction of individual polyphenolic compounds and plant extracts with biological membrane has not been fully elucidated, which prompted the authors to undertake research in this area. The aim of the study was to determine changes incurred by polyphenol compounds from blueberry fruit of the genus Vaccinium in lipid model membranes. The effects of plant extracts on model membranes were studied by means of differential scanning calorimetry (DSC), steady-state fluorimetry and attenuated total reflection Fourier transform infrared spectroscopy (ATRFTIR).

\section{Materials and methods}

\section{Materials}

The fluorescent probes 6-dodecanoyl-2-dimethylaminonaphthalene (Laurdan) and 1,6-diphenyl-1,3,5-hexatriene (DPH) were purchased from Molecular Probes, Eugene, Oregon, USA. The fluorescent probe $N, N$-dimethyl-6-propionyl-2-naphthylamine (Prodan) and the lipids 1,2-dipalmitoyl-sn-glycero-3-phosphatidylcholine (DPPC) and egg yolk lecithin (EPC) were purchased from SigmaAldrich, Steinheim, Germany.

Highbush blueberries (HB) (V. corymbosum L.) were obtained from commercial plantation from Szczecin, Poland, and lowbush blueberries (LB) (V. angustifolium L.) were collected from Experimental Station at Rajkowo near Szczecin. Fruits of wild bilberry (WB) (V. myrtillus L.) were harvested in a coniferous forest near Szczecin. Plant extracts of blueberries were prepared in the Department of Fruit, Vegetable and Plant Nutraceutical Technology of Wroclaw University of Environmental and Life Sciences.

\section{Methods}

\section{Fluidity and packing arrangement of the membranes (fluorescence spectroscopy)}

The effect of WB, HB and LB extracts on packing arrangement and fluidity of lipids in the model lipid membrane (DPPC and egg phosphatidylcholine (EPC) liposomes) was investigated using the fluorimetric method described earlier in Bonarska-Kujawa et al. [22, 23] with minor modifications. Small unilamellar liposomes (SUVs) used for fluorimetric experiments were composed of DPPC or EPC lipids. The lipids were dissolved in a chloroform/ methanol (1:1) solvent and evaporated to dryness under nitrogen. Subsequently, a phosphate buffer of $\mathrm{pH} 7.4$ was added to the obtained film and multilamellar liposomes (MLVs) were formed by mechanical shaking. Then, SUVs were formed by the sonication of DPPC dispersion in a buffer for $15 \mathrm{~min}$ at $20 \mathrm{kHz}$ using Sonics VCX750 sonicator (Sonics and Materials, Inc.). Control samples contained only lipid suspension with fluorescent probes (concentration of fluorescent probes-1 $1 \mu \mathrm{M}$ ). Extracts from $\mathrm{WB}, \mathrm{HB}$ and $\mathrm{LB}$ at a concentration of $0.005-0.05 \mathrm{mg} \mathrm{mL}^{-1}$ were added to the remaining samples. Fluorescence intensity was measured at the temperature of $37^{\circ} \mathrm{C}$ by using Laurdan and DPH fluorescent probes concentration of which was $1 \mu \mathrm{M}$. The measurements were taken with a fluorimeter (Cary Eclipse, Varian) equipped with a Peltier temperature controller DBS (temp. accuracy $\pm 0.1^{\circ} \mathrm{C}$ ). The excitation and emission wavelengths for DPH were $\lambda_{\mathrm{ex}}=360 \mathrm{~nm}, \lambda_{\mathrm{em}}=425 \mathrm{~nm}$, respectively. The excitation wavelength for Laurdan was $360 \mathrm{~nm}$, and the emitted fluorescence was recorded at two wavelengths of 440 and $490 \mathrm{~nm}$.

On the basis of the measured fluorescence intensity of probes, the values of fluorescence anisotropy $(A)$ for DPH probe and generalized polarization (GP) for the Laurdan probe were calculated using the formula described by Parasassi [24].

\section{Temperature of phase transition of DPPC (DSC and fluorescence spectroscopy)}

Samples for DSC consisted of multilamellar vesicles (MLVs) made of DPPC and modified with WB, HB and LB extracts in accordance with the procedure described earlier [23, 25]. The measurements were taken on 34-36 mg samples using Mettler Toledo Thermal Analysis System D.S.C., $821^{\mathrm{e}}$, operated at scanning speed of $2{ }^{\circ} \mathrm{C} \min ^{-1}$. An empty crucible was used as a reference, and the temperature scale was calibrated with indium.

Samples for steady-state fluorimetry spectroscopy consisted of SUVs composed of DPPC. Fluorescent probes (Prodan and DPH) were added to SUVs; then, samples were incubated for $15 \mathrm{~min}$ in darkness at room temperature. Control samples contained lipid suspension with a suitable fluorescent probe (concentration of $1 \mu \mathrm{M}$ ). Extracts from $\mathrm{WB}, \mathrm{HB}$ and $\mathrm{LB}$ at a appropriate concentration $\left(0.05 \mathrm{mg} \mathrm{mL}^{-1}\right)$ were added to the remaining samples. The measurements were taken at different temperatures above and below the main phase transition of 
DPPC. Thermal cycles $\left(20-50{ }^{\circ} \mathrm{C}\right)$ were repeated three times. GP for the Prodan probe was calculated using the formula described by Parasassi [24].

\section{Hydration of lipid membrane (ATR-FTIR)}

The experiments were made with EPC and DPPC liposomes (MLVs). IR spectra of $70 \mathrm{mM}$ liposome suspension were taken. Liposomes were prepared by the standard shaking method. Extract concentration was $0.05 \mathrm{mg} \mathrm{mL}^{-1}$. The preparations were intensively shaken with a vortex under nitrogen at room temperature with EPC and at $45{ }^{\circ} \mathrm{C}$ with DPPC liposomes. The measurements were taken using a Thermo Nicolet 6700 MCT with ZnSe crystal at room temperature. Each single spectrum was obtained from 128 records at $2 \mathrm{~cm}^{-1}$ resolution in the range of $700-4000 \mathrm{~cm}^{-1}$. Preliminary elaboration of a spectrum was done using the EZ OMNIC v 8.0 program, also of the Thermo Nicolet make. After filtering out the noise from the spectrum, the spectrum of the buffer solution was subtracted in order to remove the $\mathrm{OH}$ band of water and the baseline was corrected.

In spectra thus prepared we examined four bands: vibrations of $\mathrm{CH}_{2}$ and $\mathrm{CH}_{3}$ groups of alkyl chains-located in the range of $3000-2800 \mathrm{~cm}^{-1}$, vibrations of the carbonyl group $(\mathrm{C}=\mathrm{O})$-located in the range of $1780-1700$ and $1300-1200 \mathrm{~cm}^{-1}[26-30]$.

\section{Statistical analysis}

Statistical analysis was carried out using Statistica 9.0 (StatSoft Inc.). All the experiments were performed at least thrice unless otherwise specified. Analysis of variance was carried out, and significance between means was determined using Dunnett's post hoc test. Results are presented as mean $\pm \mathrm{SD}$. Significance levels were defined at $p<0.05$.

\section{Results}

\section{Fluidity and packing arrangement of the membrane}

The effect of WB, HB, LB extracts on fluidity of different lipid phases of liposomes formed of DPPC and EPC was studied on the basis of the fluorescence anisotropy (A) measured with the fluorescence probe DPH, which locates itself in the hydrophobic region of lipid bilayers. The DPH steady-state anisotropy is primarily related to the rotational motion restriction due to the hydrocarbon chain packing order. Therefore, the decrease in this parameter can be explained by a structural perturbation of the bilayer's hydrophobic region due to the incorporation of investigated compounds. The results of the DPH probe fluorescence anisotropy for such a membrane are presented in Table 1. No changes were observed for DPPC and EPC membranes affected by HB and LB extracts; however, a slight increase in anisotropy was observed for WB extract. The results indicate that the investigated extracts did not alter fluidity of the lipid membrane in the region occupied by the acyl chains of lipid molecules.

The degree of packing order of the hydrophilic part of membrane was investigated using the Laurdan probe. The calculated values of GP decreased with increasing HB and LB extract concentration, while the WB extract caused a slight increase in the value of GP (Table 2). This result indicates that $\mathrm{HB}, \mathrm{LB}$ and $\mathrm{WB}$ extracts change the arrangement of the hydrophilic region of the lipid membrane, probably through penetration into this area or through absorption on the surface of the membrane.

\section{Temperature of phase transition of lipid membranes}

DSC and steady-state fluorimetry were used to study the effect of WB, HB and LB extracts on phase transitions of DPPC membranes. The above-mentioned methods were employed to systematically monitor the influence of the extract on different membrane regions. In particular, the calorimetric measurements helped to analyze the effect of tested extracts on the temperature of the pretransition $\left(T_{\mathrm{p}}\right)$ and the main transition $\left(T_{\mathrm{m}}\right)$ of DPPC. Phospholipids show thermotropic and lyotropic phase behavior. These lipids form hydrated biomolecular lamellar phases, in which the lipid molecules are packed either in a quasi-crystalline twodimensional lattice (gel phase) or remain in a lamellar arrangement but show higher two-dimensional fluidity. Transition between these phases can be induced by temperature changes. The main phase transition $\left(T_{\mathrm{m}}\right)$ is from the lamellar gel phase to liquid (fluid) phase; however, DPPC shows a more complicated phase behavior: There is also so-called intermediate (ripple) $P_{\beta^{\prime}}$ phase, in which the surface of the bilayer is distorted by a periodic ripple, and $L_{\beta^{\prime}}$ phase in which the molecules are tilted at an angle of about $30^{\circ}$ relative to bilayer normal. The change from $L_{\beta^{\prime}}$ to $P_{\beta^{\prime}}$ is commonly referred to as the pretransition $\left(T_{\mathrm{p}}\right)$ [22-25, 31-35].

The DSC measurements were taken for a number of selected concentrations of extracts within a very wide range: $0.05-10 \mathrm{mg} \mathrm{mL}^{-1}$ (Fig. 1). Even at the highest concentrations of $\mathrm{WB}, \mathrm{LB}$ and $\mathrm{HB}$ extracts $\left(10 \mathrm{mg} \mathrm{mL}^{-1}\right)$, we did not observe any significant changes in $T_{\mathrm{m}}$ (control $41.0 \pm 0.2{ }^{\circ} \mathrm{C}, \mathrm{WB} 40.3 \pm 0.2{ }^{\circ} \mathrm{C}, \mathrm{HB} 41.0 \pm 0.2{ }^{\circ} \mathrm{C}, \mathrm{LB}$ $40.7 \pm 0.2^{\circ} \mathrm{C}$; Fig. 1c). Also the half width of the peak $\left(\Delta T_{1 / 2}\right)$ did not change for LB and HB extract (control 
Table 1 Values of anisotropy $(A)$ of the DPH probe for liposomes formed from DPPC and EPC modified with the extracts from wild blueberry (WB), high blueberry (HB) and low blueberry (LB) at $37^{\circ} \mathrm{C}$

\begin{tabular}{|c|c|c|c|c|c|c|}
\hline \multirow[t]{2}{*}{$\mathrm{mg} \mathrm{mL}^{-1}$} & \multicolumn{6}{|l|}{$A \pm \mathrm{SD}$} \\
\hline & $\begin{array}{l}\text { DPPC } \\
\text { WB }\end{array}$ & EPC & $\begin{array}{l}\text { DPPC } \\
\text { HB }\end{array}$ & EPC & $\begin{array}{l}\text { DPPC } \\
\text { LB }\end{array}$ & EPC \\
\hline 0.0000 & $0.222 \pm 0.009$ & $0.071 \pm 0.002$ & $0.222 \pm 0.009$ & $0.071 \pm 0.002$ & $0.222 \pm 0.009$ & $0.071 \pm 0.002$ \\
\hline 0.0050 & $0.228 \pm 0.004$ & $0.076 \pm 0.002$ & $0.228 \pm 0.005$ & $0.067 \pm 0.002$ & $0.216 \pm 0.002$ & $0.069 \pm 0.002$ \\
\hline 0.0075 & $0.230 \pm 0.003$ & $0.076 \pm 0.010$ & $0.226 \pm 0.002$ & $0.068 \pm 0.002$ & $0.222 \pm 0.003$ & $0.069 \pm 0.003$ \\
\hline 0.0100 & $0.233 \pm 0.006$ & $0.089 \pm 0.004$ & $0.226 \pm 0.006$ & $0.067 \pm 0.002$ & $0.224 \pm 0.004$ & $0.069 \pm 0.001$ \\
\hline 0.0250 & $0.233 \pm 0.004$ & $0.085 \pm 0.012$ & $0.227 \pm 0.005$ & $0.069 \pm 0.002$ & $0.223 \pm 0.005$ & $0.070 \pm 0.002$ \\
\hline 0.0500 & $0.238 \pm 0.005$ & $0.091 \pm 0.006$ & $0.228 \pm 0.004$ & $0.070 \pm 0.002$ & $0.225 \pm 0.002$ & $0.071 \pm 0.003$ \\
\hline
\end{tabular}

Table 2 Values of generalized polarization (GP) of the Laurdan probe for liposomes formed from DPPC and EPC modified with the extracts from wild blueberry (WB), high blueberry (HB) and low blueberry (LB) at $37{ }^{\circ} \mathrm{C}$

\begin{tabular}{lllllll}
\hline $\mathrm{mg} \mathrm{mL}^{-1}$ & GP $\pm \mathrm{SD}$ & & & & \\
\cline { 2 - 6 } & DPPC & EPC & DPPC & EPC & DPPC & LB \\
& WB & & HB & & & EPC \\
\hline 0.00 & $0.187 \pm 0.038$ & $-0.341 \pm 0.008$ & $0.187 \pm 0.038$ & $-0.341 \pm 0.008$ & $0.187 \pm 0.038$ & $-0.341 \pm 0.008$ \\
0.01 & $0.159 \pm 0.011$ & $-0.339 \pm 0.001$ & $0.125 \pm 0.009$ & $-0.355 \pm 0.001$ & $0.124 \pm 0.012$ & $-0.349 \pm 0.004$ \\
0.02 & $0.175 \pm 0.001$ & $-0.325 \pm 0.003$ & $0.126 \pm 0.001$ & $-0.353 \pm 0.003$ & $0.117 \pm 0.007$ & $-0.356 \pm 0.002$ \\
0.03 & $0.191 \pm 0.004$ & $-0.310 \pm 0.005$ & $0.135 \pm 0.002$ & $-0.359 \pm 0.001$ & $0.122 \pm 0.003$ & $-0.356 \pm 0.006$ \\
0.04 & $0.181 \pm 0.044$ & $-0.301 \pm 0.005$ & $0.130 \pm 0.008$ & $-0.358 \pm 0.003$ & $0.118 \pm 0.009$ & $-0.359 \pm 0.001$ \\
0.05 & $0.220 \pm 0.004$ & $-0.301 \pm 0.016$ & $0.132 \pm 0.003$ & $-0.360 \pm 0.004$ & $0.119 \pm 0.006$ & $-0.361 \pm 0.005$ \\
\hline
\end{tabular}

$\left.0.7 \pm 0.2{ }^{\circ} \mathrm{C}, \quad \mathrm{HB} \quad 0.8 \pm 0.2{ }^{\circ} \mathrm{C}, \quad \mathrm{LB} \quad 0.86 \pm 0.2^{\circ} \mathrm{C}\right)$ although it increased slightly for $\mathrm{WB}$ extract (control $0.7 \pm 0.2{ }^{\circ} \mathrm{C}$, WB $1.26 \pm 0.2{ }^{\circ} \mathrm{C}$ ). The extracts did not change $T_{\mathrm{p}}$ and $T_{\mathrm{m}}$ of a model membrane formed with DPPC, which suggests that their components do not induce significant changes in liquidity of the bilayer or the membrane structure. Increased concentration of the extracts caused only a reduced heat effect originating from the DPPC pretransition, until it completely disappeared, which may suggest that the tested compounds cause only small changes in the polar part of the membrane.

The phase transitions and the fluidity of the lipid bilayer at different temperatures were determined using fluorescence probes located in hydrophobic regions (DPH probe) or hydrophilic (Prodan probe). Using the DPH probe, we assessed how the fluidity of the model membrane modified with $\mathrm{HB}, \mathrm{LB}$ and $\mathrm{WB}$ extracts changes with the temperature. The dependence of DPH anisotropy $(A)$ on temperature for DPPC liposome membranes is presented in Fig. 2a. The LB and HB extract $\left(0.05 \mathrm{mg} \mathrm{mL}^{-1}\right)$ caused a slight increase in the value of $A$, both in a gel (below $T_{\mathrm{m}}=41{ }^{\circ} \mathrm{C}$ ) and liquid-crystal (above $T_{\mathrm{m}}$ ) phase of DPPC model membrane. Hence, LB and HB slightly decrease the liquidity of the hydrophobic part of the bilayer, but do not significantly change the $T_{\mathrm{m}}$ of DPPC. The WB extract $\left(0.05 \mathrm{mg} \mathrm{mL}^{-1}\right)$ decreased slightly the fluidity of the membrane in the gel phase and increased it in the liquidcrystal phase, without changing the $T_{\mathrm{m}}$ of DPPC membrane. Thus, in the presence of each of tested extracts, we did not observe significant changes in anisotropy with changing temperature, which indicates that compounds from extracts interact with the membrane only at its surface.

The packing order of the hydrophilic region of lipid membranes formed of DPPC in correlation to temperature was investigated by using Prodan probe. GP of Prodan in SUVs formed of DPPC is presented in Fig. 2b. The presence of the extracts at a concentration of $0.05 \mathrm{mg} \mathrm{mL}^{-1}$ did not result in substantial changes of $T_{\mathrm{m}}$ of DPPC. HB and LB extracts slightly increased the value of GP in the gel phase of DPPC, but slightly decreased it in the liquid phase. An increase in GP was observed in the presence of the WB extract, both in the gel and in the liquid phase of DPPC. 
Fig. 1 Calorimetric curves of DPPC MLVs in the presence of extracts from the fruits of high blueberries (a) and wild blueberries (b). The curves are normalized for the amount of DPPC; scan rates $2{ }^{\circ} \mathrm{C} \mathrm{min}-1$

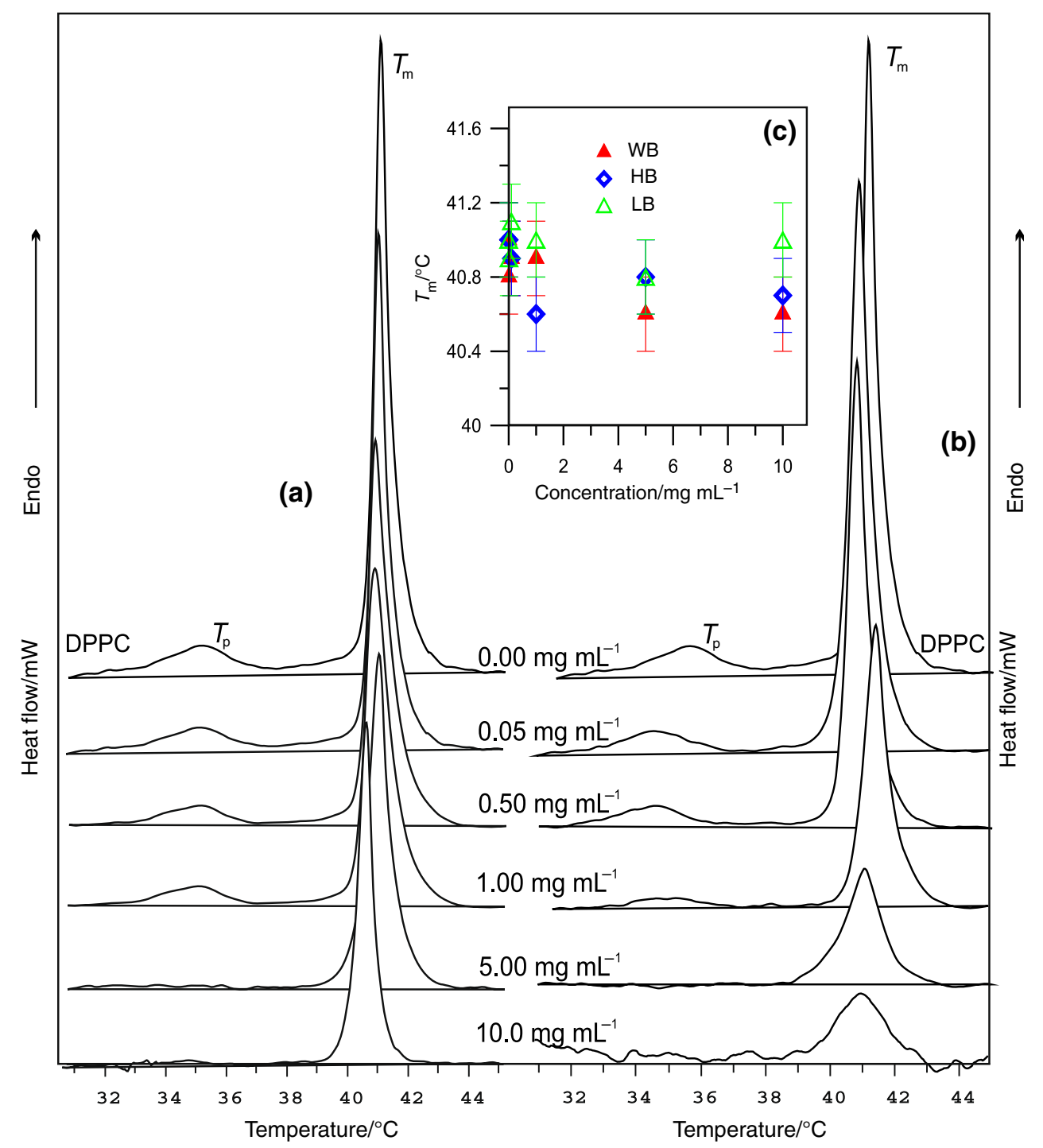

\section{Hydration of lipid membranes}

Using the ATR-FTIR methods, we examined changes of individual structural elements of liposome membranes formed of DPPC and EPC in the presence of extracts from the fruits of lowbush, highbush and wild blueberry. No effects of tested extracts, at any of the concentrations used, were observed in any spectra reflecting changes in the hydrophobic part of the lipid membrane. WB, HB and LB extracts did not cause meaningful changes in the polar part of the membrane, whereas they caused slight changes in the phosphate and choline band of spectra, indicating a weak interaction of these compounds with the surface part of the membrane.

Figure 3 shows the choline ( $\left.3 a, 3 a^{\prime}\right)$, phosphate $\left(3 b, 3 b^{\prime}\right)$ and carbonyl $\left(3 \mathrm{c}, 3 \mathrm{c}^{\prime}\right)$ bands of spectra for DPPC and EPC suspensions in the presence of the $0.05 \mathrm{mg} \mathrm{mL}^{-1}$ concentration of $\mathrm{WB}, \mathrm{HB}$ and $\mathrm{LB}$ extracts. A marked reduction in the width at half maximum of the choline band of liposomes formed of DPPC can be observed in the presence of tested extracts, especially extracts of WB and HB (Fig. 3a). Similar changes were observed for EPC membranes (Fig. 3a').

Figure $3 \mathrm{~b}$ shows the phosphate band of DPPC liposomes. At room temperatures, the membrane is in the gel phase and the maximum band is achieved for the wave number $1225 \mathrm{~cm}^{-1}$. The presence of the extracts slightly changed the relative intensity of various parts of the band. Extracts from the WB decrease intensity of the component of the wave number $1222 \mathrm{~cm}^{-1}$, and the LB and HB extracts did not change this wave number. Figure $3 b^{\prime}$ shows the phosphate band of EPC liposomes. Their membranes at room temperature are in the liquid-crystalline phase. There are no meaningful differences between 


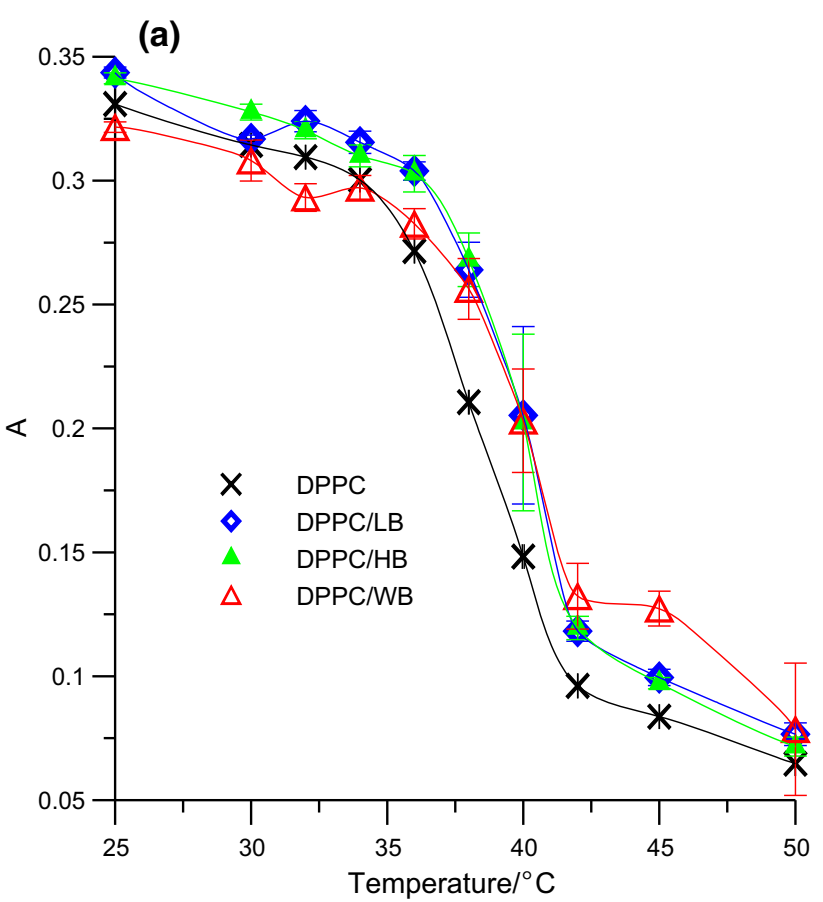

(b)

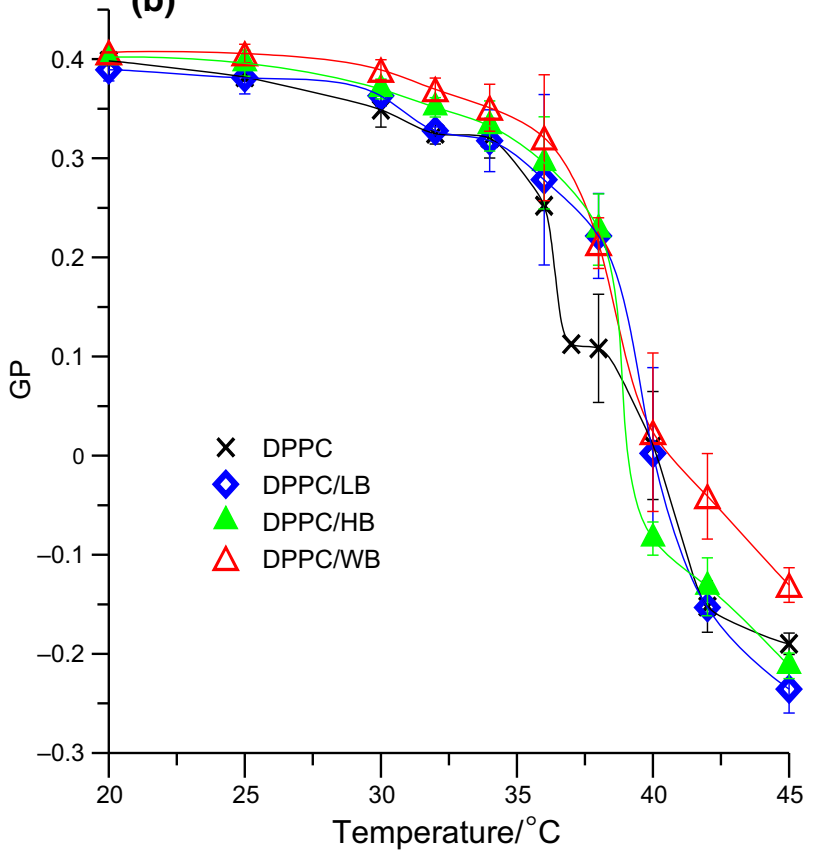

Fig. 2 Values of anisotropy (a) for DPH probes and values of GP for Prodan (b) at different temperatures for DPPC liposomes (SUVs) with tested compounds added at $0.05 \mathrm{mg} \mathrm{mL}^{-1}$

the control probe and EPC with fruits extracts added, but the whole band is slightly shifted toward lower wave numbers. These changes indicate that the degree of hydration of the phosphate groups of the membranes increased due to the presence of extracts.
Fig. 3 ATR-FTIR spectra of the DPPC/extracts $(\mathbf{a}, \mathbf{b}, \mathbf{c})$ and of the EPC/extracts $\left(\mathbf{a}^{\prime}, \mathbf{b}^{\prime}, \mathbf{c}^{\prime}\right)$ measured at room temperature; $\mathbf{a}, \mathbf{a}^{\prime}$ choline band of lecithin; $\mathbf{b}, \mathbf{b}^{\prime}$ phosphate band of lecithin; $\mathbf{c}, \mathbf{c}^{\prime}$ carbonyl band of lecithin

\section{Discussion}

The knowledge how the polyphenolic extracts interact with the cell membrane and mechanisms responsible for their protective effect on the organism at the molecular level is crucial for dietetics and pharmacy. In the manuscript [36], the authors determined the content of polyphenolic compounds in the bilberry extracts: The most predominant were the anthocyanin derivatives which constituted $37.49 \%$ of fruit extract in high blueberries, $25.90 \%$ in low blueberries and $63.97 \%$ in wild blueberries. The main polyphenolic components of the studied fruit extracts were the following anthocyanins: derivatives of delphinidin and derivatives of cyanidin and small amount of derivatives of malvidine, peonidin, petunidin. Anthocyanin dominated in WB extract, while LB and HB extracts also contained chlorogenic acid, which constituted about $30 \%$ of identificated compounds [36].

Interaction of the extracts with the lipid model membranes was determined on the basis of physical changes incurred in the membranes, using the following techniques: calorimetric, fluorimetric and ATR-FTIR. The effects of extracts' polyphenolic compounds on the membranes were determined on the basis of degree of the order of the polar heads of lipids, membrane fluidity, degree of membrane hydration and changes in phase transition temperature of model lipid membranes.

Using fluorescent probes: DPH, Prodan and Laurdan, the effect of extracts on the properties of the hydrophilic and hydrophobic region of membranes was examined. Modification of both those parts affects the intensity of fluorescence emitted by probes located in the respective areas and, consequently, the calculated values of fluorescence anisotropy $(A)$ or generalized polarization (GP). The fluidity of membranes in the hydrophobic region, i.e., at the level of alkyl chains, was established on the basis of fluorescence anisotropy of the DPH probe. Changes in fluorescence anisotropy of the probe reflect mobility of the marker in the membrane, which depends on the movement and arrangement of lipid molecules. DPH probe emits fluorescence from the hydrophobic part of the membrane, where the alkyl chains of membrane lipids are located. The studies of membranes fluidity have shown that polyphenolic blueberries' extracts do not result in a significant change of the fluorescence anisotropy of DPH probe, and therefore do not alter substantially fluidity of all used model membranes in the hydrocarbon chains region $[24,36]$. Only the wild 

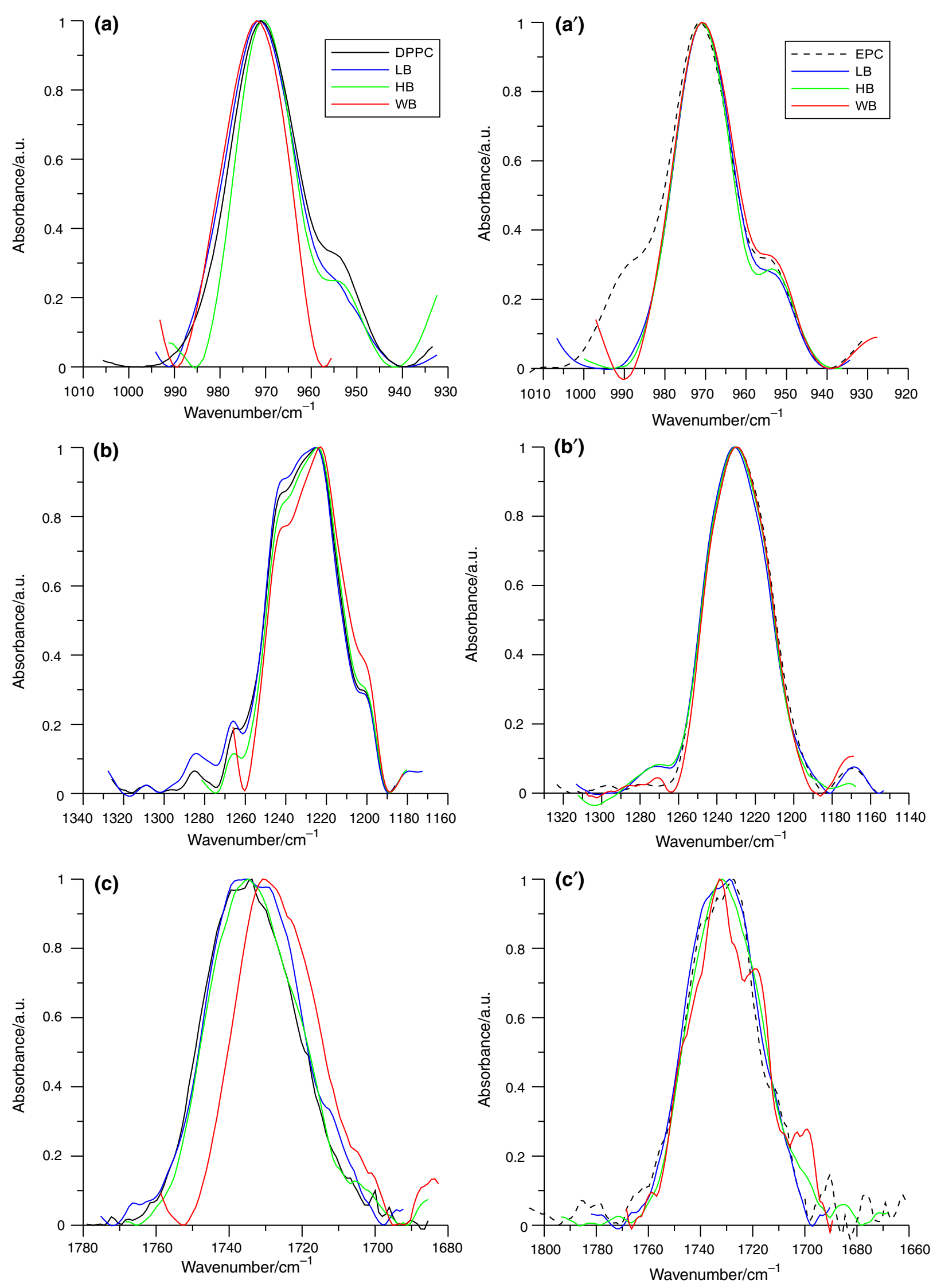
blueberry extract (WB) caused small changes in this region of EPC model membrane and slightly decreased membrane fluidity. The results of the study indicate that the tested extracts practically do not change the fluidity of membranes. One can thus conclude that polyphenolics bind to the surface of membranes, interacting with them electrostatically, and that they either do not penetrate to the hydrophobic area of membrane or do that in small quantities.

Changes in the hydrophilic part of membranes under the action of the tested substances were determined on the basis of GP of Laurdan probe the chromophore of which is incorporated at the level of glycerol. The GP parameter depends on polarity of the chromophore environment-GP changes indicate changes in the polar heads of the lipid membrane. The decline in the value of the GP in the presence of modifying substances testifies to the growth of disorder in the polar heads of lipids and their greater dynamics, whereas GP growth is associated with increased order and lower dynamics of the polar heads of lipids [24]. Studies with the use of this probe showed significant changes of order in the hydrophilic part in the presence of polyphenolic substances. The decline in the value of the GP means that the substances contained in the extract cause an increase in the disorder of the hydrophilic area of the lipid bilayer. Additionally, the GP value decreased with increasing concentrations of the extracts, i.e., with the amount of polyphenolic compounds that are attached to the hydrophilic area of the membranes [22,36]. In the presence of HB and LB extract, a concentration-dependent decrease in GP of Laurdan probe occurs. This result indicates that $\mathrm{HB}, \mathrm{LB}$ and WB extracts change the arrangement of the hydrophilic region of the liposome membranes, probably through penetration into this area or absorption on the surface of the membrane.

Phase transition temperature study was conducted for the membranes created from DPPC lipids in different temperatures using DSC and steady-state fluorimetric spectroscopy. The effect of polyphenolic compounds on the thermodynamic parameters of multilayer liposomes was established. Using fluorimetric method, the thermotropic parameters of fluorescence anisotropy and generalized polarization were determined. For the membranes created from one type of lipid molecules, it is possible to determine the transition temperature from the gel phase, where the lipid molecules are packed tighter and exhibit less hydrocarbon chains mobility, to the liquid-crystalline state with much greater mobility and lesser compression of the molecules [37]. Fluorimetric study was carried out using the hydrophobic fluorescent probe DPH and the hydrophilic probe Prodan. The study was performed at different temperatures for SUVs created with DPPC and treated with selected concentrations of fruit extracts of lowbush, highbush and wild bilberry [22, 36, 38, 39]. Research conducted with the use of DPH probe has shown that tested substances neither change the main phase transition temperature nor fluorescence anisotropy, and thus the fluidity of membranes in the liquid-crystalline phase, as well as in the gel phase, compared with modified liposomes $[38,39]$. Extract of wild bilberry decreased slightly membrane fluidity in the gel phase, but increased it in the liquidcrystalline state. The results obtained for the hydrophilic probe Prodan exhibit thermotropic changes in generalized polarization of membranes modified with polyphenolic substances. The chromophore of Prodan probe is located above glycerol, at the border between the hydrophilic region and the water environment, and is sensitive to changes in hydration and packing of lipid molecules, as well as to changes of the temperature of the pretransition that characterizes the transitional phase between the liquidcrystalline and gel phase [24]. LB and HB extracts caused the value of the GP of Prodan probe in gel phase to remain unchanged, but increased it in the liquid-crystalline phase, without altering the main phase transition temperature. Similarly, the results for WB extract revealed changes both in the gel- and liquid-crystalline phases, where GP value increased, without altering the main phase transition temperature, but with changes in the transitional region. These results indicate that the extract's polyphenols do not alter the structure of the membrane and do not penetrate into the hydrophobic interior of the membrane. However, they associate with its surface changing the packing of the lipid polar heads and their dynamics, i.e., change the packing arrangement inside membranes, in the region of both the gel phase and in the liquid-crystalline phase. The authors Kużdżał et al. [40] suggest that increase in GP value could be connected with decreasing amounts of water molecules bonded with the membrane's surface, around the chromophore of the probe.

In order to confirm the results obtained in the fluorimetric experiments, we carried out studies using DSC. Changes of the main phase transition temperature $\left(T_{\mathrm{m}}\right)$ caused by the presence of additional compounds in the membrane are associated with changes in fluidity of the lipid bilayer and changes of the membrane structure. Pretransition temperature $\left(T_{\mathrm{p}}\right)$ changes provide evidence for changes rather in the polar part of the membrane that result in loosening its structure [37, 41, 42]. Calorimetric studies were carried out with the membrane model in the form of MLVs formed of DPPC modified with the extracts and polyphenolic compounds [22, 36-39]. Calorimetric test results showed little change in $T_{\mathrm{m}}$ only for very large concentrations of polyphenolic substances $\left(10 \mathrm{mg} \mathrm{mL}^{-1}\right)$, whereas for lower concentrations virtually no change was found. To a small extent, the polyphenols changed $T_{\mathrm{p}}$ in the lipid membranes, removing it at the highest concentrations 
and slightly increasing the half width of the main phase transition peak $\left(\Delta T_{1 / 2}\right)$. Lack of effect of polyphenolic substances on the main phase transition temperature shows that they do not change the structure and organization of the lipid bilayer, without penetrating deeply into the hydrophobic region of membranes, and the changes in pretransition temperature and cooperativity of the peak attest to the presence of the test substances on the surface of the membrane. There was also an increase in the peak cooperativity and in phase transition enthalpy. This result confirms that the compounds do not change the structure of the membrane, but modify its hydrophilic part through electrostatic interactions with the polar heads of lipids.

In order to assess changes in the hydrophilic part of membrane modified with the extracts and polyphenolic compounds, measurements of the degree of membrane hydration were made by using the ATR-FTIR method. Changes in the hydration of the DPPC or EPC membrane surface were induced by the presence of polyphenolic substances in it. In the hydration studies, based on obtained IR spectra, the following four bands were taken into account: $3000-2800 \mathrm{~cm}^{-1}$ - vibrations of methylene and methyl groups $\left(\mathrm{CH}_{2}\right.$ and $\left.\mathrm{CH}_{3}\right)$ of alkyl chains; $1780-1700 \mathrm{~cm}^{-1}-$ vibrations of carbonyl groups $(\mathrm{C}=\mathrm{O}) ; 1300-1200 \mathrm{~cm}^{-1}$ phosphate groups $\left(\mathrm{PO}_{2}\right) ; 1000-900 \mathrm{~cm}^{-1}$ - choline groups $\left(\mathrm{N}-\mathrm{CH}_{3}\right)$. The frequency of vibration of methylene and methyl groups of the alkyl chains depend on mobility (fluidity) of the chains and grow, for example, with temperature or during transition from the liquid-crystalline to gel state. The increase in the wave number of these bands testifies to an increase in fluidity of the hydrophobic region of the membrane. Carbonyl groups, and even more so-phosphate groups, form hydrogen bonds with the water. Carbonyl group can bind one molecule of water, the phosphate group a few of them. Therefore, the carbonyl and phosphate band of phospholipids corresponds to the sum of the vibrations of the groups $\mathrm{C}=\mathrm{O}$ or $\mathrm{PO}_{2}$ which are in varying degrees of hydration. Vibrations of the groups $\mathrm{C}=\mathrm{O}$ and $\mathrm{PO}_{2}$ that do not bind water correspond to, respectively, wave numbers at $1740 \mathrm{~cm}^{-1}$ and at $1260 \mathrm{~cm}^{-1}$. Each bound water molecule moves these values by about $20 \mathrm{~cm}^{-1}$ in the direction of smaller numbers. The changes observed in these bands are evidence of changes in the degree of hydration of the membranes in the region of carbonyl and phosphate groups. The frequencies of vibration of the methyl groups of the choline part depend on the properties of the surrounding area and grow slightly with decreasing viscosity. The wave number for vibrations of completely dry lipid is just over two units less than that of completely hydrated lipid membrane (a liposome). The half width of the band depends on the "stiffness" of the polar head, namely on the capacity to move along the skeleton $-\mathrm{N}-\mathrm{C}-\mathrm{C}-\mathrm{O}-\mathrm{P}-\mathrm{O}-$ [28-30].
Using the IR, we examined changes of individual structural elements of liposome membranes formed of DPPC and egg lecithin (EPC) in the presence of extracts from the fruits of HB, LB and WB [36]. The investigation showed that these extracts did not cause changes in the hydrophobic part of liposome membranes, in the frequency range corresponding to vibrations of the bonds of $\mathrm{CH}_{3}$ and $\mathrm{CH}_{2}$ in the hydrocarbon chains of lipid molecules. However, minor shifts in the carbonyl, choline and phosphate bands of DPPC were observed in the absorption IR spectra: smaller for the LB and HB extracts and slightly greater for the WB. However, the nature of the changes in the hydrophilic part of the liposome membrane, reflected in the slightly amended shape of the spectrum with an additional maximum of the carbonyl band, pointed out the lack of direct bindings between polyphenolic compounds of the extracts and carbonyl groups of lipids. The observed changes may be the result of the formation of hydrogen bonds between the $\mathrm{OH}$ groups of polyphenols and oxygen in the ester binding of lipids, which was also suggested by other authors [43]. In the parts of the spectrum corresponding to the vibrations of the phosphate groups, we also observed a shift of the maximum toward smaller frequencies that was larger for wild blueberry extracts, indicating greater hydration under the influence of these extracts. In addition, in the choline band a reduction in the peak half width was evidenced. The shape of the spectrum in the choline band indicates limitations in the movement of the choline group as a result of interactions with molecules of the extracts. These results indicate that the polyphenols are likely to make bonds with the polar heads of lipids, which would limit their mobility in the area of the choline group, and suggest an increase in the concentration of water molecules in the polar region of the membrane.

\section{Conclusions}

The research presented in the scientific achievement was to determine changes in the physical properties of lipid membranes induced by the studied extracts of highbush, lowbush and wild bilberry and specify the location of the substances in the areas of membrane modified by them.

All experimental results indicate that polyphenol compounds contained in the lowbush, highbush and wild bilberry interact with polar part of the surface of the membrane and had practically no influence on fluidity in the hydrophobic region on the membranes. Polyphenolic compounds created specific protective coat around the lipid membrane, through the location on the membrane surface.

Minor differences between interacting polyphenolic extracts with three ecotypes of Vaccinum bilberry with 
model membrane could be result of different quality contents of the extract.

Acknowledgements The use of a DSC in the Institute of Agricultural Engineering of Wrocław University of Environmental and Life Sciences is gratefully acknowledged. Hanna Pruchnik acknowledges financial support of the statutory activities of the Department of Physics and Biophysics of the Wrocław University of Environmental and Life Sciences.

Open Access This article is distributed under the terms of the Creative Commons Attribution 4.0 International License (http://creative commons.org/licenses/by/4.0/), which permits unrestricted use, distribution, and reproduction in any medium, provided you give appropriate credit to the original author(s) and the source, provide a link to the Creative Commons license, and indicate if changes were made.

\section{References}

1. Saponjac VT, Canadanovic-Brunet J, Cetkovic G, Djilas S, Cetojevic-Simin D. Dried bilberry (Vaccinium myrtillus L.) extract fractions as antioxidants and cancer cell growth inhibitors. LWT. 2015;61:615-21.

2. Kim J, Lee K, Lee H, Watson RR, Preedy VR, Zibadi S. Polyphenols suppress and modulate inflammation. Polyphen Hum Health Dis. 2014;1:393-408.

3. Rodrigo R, Gil D, Miranda Merchak A, Kalantzidis G. Antihypertensive role of polyphenols. Adv Clin Chem. 2012;58:225-54.

4. Silva S, Costa EM, Pereira MF, Costa MR, Pintado ME. Evaluation of the antimicrobial activity of aqueous extracts from dry Vaccinium corymbosum extracts upon food microorganism. Food Control. 2013;34:645-50.

5. Daglia M. Polyphenols as antimicrobial agents. Curr Opin Biotechnol. 2012;23:174-81.

6. Oprea E, Ruta LL, Nicolau I, Popa CV, Neagoe AD, Farcasanu IC. Vaccinium corymbosum L., (blueberry) extracts exhibit protective action against cadmium toxicity in Saccharomyces cerevisiae cells. Food Chem. 2014;152:516-21.

7. Shen X, Sun X, Xie Q, Liu H, Zhao Y, Pan Y, Hwang C, Wu $\mathrm{VCH}$. Antimicrobial effect of blueberry (Vaccinium corymbosum L.) extracts against the growth of Listeria monocytogenes and Salmonella Enteritidis. Food Control. 2014;35:159-65.

8. Paller CJ, Paller X, Ye PJ, Wozniak BK, Gillespie PR, Sieber $\mathrm{RH}$, Carducci R. A randomized phase II study of pomegranate extract for men with rising PSA following initial therapy for localized prostate cancer. Prostate Cancer Prostatic Dis. 2013;16:50-5.

9. Adams LS, Kanaya N, Phung S, Liu Z, Chen S. Whole blueberry powder modulates the growth and metastasis of MDA-MB-231 triple negative breast tumors in nude mice. J Nutr. 2011;141:1805-12.

10. Basu A, Du M, Leyva MJ, Sanchez K, Betts NM, Wu M, Aston $\mathrm{CE}$, Lyons TJ. Blueberries decrease cardiovascular risk factors in obese men and women with metabolic syndrome. J Nutr. 2010;140:1582-7.

11. Bomser JA, Madhavi DL, Singletary K, Smith MAL. Induction of $\mathrm{NAD}(\mathrm{P}) \mathrm{H}$-(quinone-acceptor) oxidoreductase in murine hepatoma-cells (Hepa 1c1c7) by fruit (Vaccinium) extracts. Fed Am Soc Exp Biol J. 1995;9:993.

12. Bornsek SM, Ziberna L, Polak T, Vanzo A, Ulrih NP, Abram V, Tramer F, Passamonti S. Bilberry and blueberry anthocyanins act as powerful intracellular antioxidants in mammalian cells. Food Chem. 2012;134:1878-84.

13. Gong P, Chen F, Wang L, Wang J, Jin S, Ma Y. Protective effects of blueberries (Vaccinium corymbosum L.), extract against cadmium-induced hepatotoxicity in mice. Environ Toxic Pharmacol. 2014;37:1015-27.

14. Smith MAL, Marley KA, Seigler D, Singletary KW, Meline B. Bioactive properties of wild blueberry fruits. J Food Sci. 2000;65:352-6.

15. Calò R, Marabini L. Protective effect of Vaccinium myrtillus extract against UVA- and UVB-induced damage in a human keratinocyte cell line (HaCaT cells). J Photochem Photobiol B Biol. 2014;132:27-35.

16. Norberto S, Silva S, Meireles M, Faria A, Pintado M, Calhau C. Blueberry anthocyanins in health promotion: a metabolic overview. J Funct Food. 2013;5:1518-28.

17. Sancho RAS, Pastore GM. Evaluation of the effects of anthocyanins in type 2 diabetes. Food Res Int. 2012;46:378-86.

18. Galvano F, La Fauci L, Lazzarino G, Fogliano V, Ritieni A, Ciappellano S, Battistini N, Tavazzi B, Galvano G. Cyanidins: metabolism and biological properties. J Nutr Biochem. 2004;15:2-11.

19. Hendrich A. Flavonoid-membrane interactions: possible consequences for biological effects of some polyphenolic compounds. Acta Pharmacol Sin. 2006;27:27-40.

20. Bukowska B, Michałowicz J, Krokosz A, Sicińska P. Comparison of the effect of phenol and its derivatives on protein and free radical formation in human erythrocytes (in vitro). Blood Cells Mol Dis. 2007;39:238-44.

21. Tarahovsky YS, Muzafarov EN, Kim YA. Rafts making and rafts braking: how plant flavonoids may control membrane heterogeneity. Mol Cell Biochem. 2008;314:65-71.

22. Bonarska-Kujawa D, Pruchnik H, Oszmiański J, Sarapuk J, Kleszczyńska $\mathrm{H}$. Changes caused by fruit extracts in the lipid phase of biological and model membranes. Food Biophys. 2011;6:58-67.

23. Pruchnik H, Włoch A, Żyłka R, Oszmiański J, Kleszczyńska H. Interaction of skullcap (Scutellaria baicalensis Georgi) and buckwheat (Fagopyrum esculentum Moench) extracts with lipid bilayers. J Therm Anal Calorim. 2015;121:475-84.

24. Parasassi T, Krasnowska EK, Bagatolli L, Gratton E. Laurdan and prodan as polarity-sensitive fluorescent membrane probes. J Fluoresc. 1998;8:365-73.

25. Pruchnik $H$. Influence of cytotoxic butyltin complexes with 2-sulfobenzoic acid on the thermotropic phase behavior of lipid model membranes. J Therm Anal Calorim. 2017;127:507-14.

26. Tien HT. Bilayer lipid membranes: theory and practice. New York: Marcel Dekker. Inc.; 1974.

27. Binder $\mathrm{H}$. The molecular architecture of lipid membranes-new insights from hydration-tuning infrared linear dichroism spectroscopy. Appl Spectrosc Rev. 2003;38:15-69.

28. Hubner $\mathrm{W}$, Mantsch $\mathrm{HH}$. Orientation of specifically ${ }^{13} \mathrm{C}=\mathrm{O}$ labeled phosphatidylcholine multilayers from polarized attenuated total reflection FT-IR spectroscopy. Biophys J. 1991;59:1261-72.

29. Lewis RNAH, McElhaney RN, Pohle W, Mantsch HH. Components of the carbonyl stretching band in the infrared spectra of hydrated 1,2-diacylglycerolipid bilayers: a reevaluation. Biophys J. 1994;67:2367-75.

30. Attar M, Kates M, Khalil MB, Carrier D, Wong PTT. A Fouriertransform infrared study of the interaction between germ-cell specific sulfogalactosylglycerolipid and dimyristoylglycerophosphocholine. Chem Phys Lipids. 2000;106:101-14.

31. Blume A. Biological calorimetry: membranes. Thermochim Acta. 1991;193:299-347. 
32. Di Foggia M, Bonora S, Tinti ATV. DSC and Raman study of DMPC liposomes in presence of Ibuprofen at different $\mathrm{pH}$. J Therm Anal Calorim. 2017;127:1407-17.

33. Owusu-Ware SK, Chowdhry BZ, Leharne SA, Antonijevic MD. Phase behaviour of dehydrated phosphatidylcholines. J Therm Anal Calorim. 2017;127:415-21.

34. Chatzigeorgiou P, Mourelatou A, Pollatos E, Margari D, Zogzas N, Viras K, Mavromoustakos T, Semidalas ChE. Comparison of the thermal behavior and conformational changes in partially and fully hydrated dipalmitoylphosphatidylcholine system. J Therm Anal Calorim. 2018;131:887-98.

35. Wei TT, Sun HY, Deng G, Gu JY, Guo HY, Xu J, Wu RG. The interaction of paeonol with DPPC liposomes. J Therm Anal Calorim. 2018;132:685-92.

36. Ochmian I, Oszmiański J, Skupień K. Chemical composition, phenolics and firmness of small black fruits. J Appl Bot Food Qual. 2009;83:64-9.

37. Bonarska-Kujawa D, Pruchnik H, Cyboran S, Żyłka R, Oszmiański J, Kleszczyńska H. Biophysical mechanism of the protective effect of the polyphenols extracts from blue honeysuckle (Lonicera caerulea L. var. kamtschatica Sevast.) against lipid peroxidation of erythrocyte and lipid membranes. J Mem Biol. 2014;247:611-25.

38. Pruchnik H, Bonarska-Kujawa D, Kleszczyńska H. Effect of chlorogenic acid on the phase transition in phospholipid and phospholipid/cholesterol membranes. J Therm Anal Calorim. 2014;118:943-50.

39. Cyboran S, Bonarska-Kujawa D, Pruchnik H, Żyłka R, Oszmiański J, Kleszczyńska H. Phenolic content and biological activity of extracts of blackcurrant fruit and leaves. Food Res Int. 2014;65:47-58.

40. Kużdżał M, Wesołowska O, Strancar J. Fluorescence and ESR spectroscopy studies on the interaction of isoflavone genistein with biological and model membranes. Chem Phys Lipids. 2011;164:283-91.

41. McMullen TPW, Lewis RNAH, McElhaney RN. Differential scanning calorimetric study of the effect of cholesterol on the thermotropic phase behaviour a homologous series of linear saturated phosphatidylcholines. Biochem. 1993;32:516-22.

42. McMullen TPW, McElhaney RN. New aspects of the interaction of cholesterol with dipalmitoylphosphatidylcholine bilayers as revealed by high-sensitivity differential scanning calorimetry. Biochim Biophys Acta. 1995;1234:90-8.

43. Pawlikowska-Pawlęga B, Misiak LE, Zarzycka B, Paduch R, Gawron A, Gruszecki WI. FTIR, ${ }^{1}$ H NMR and EPR spectroscopy studies on the interaction of flavone apigenin with dipalmitoylphosphatidylcholine liposomes. Biochem Biophys Acta. 2013;1828:518-27. 